\title{
Conductor Limited Quenches of LHC Superconducting Main Dipoles
}

\author{
M. Pojer, P. Pugnat, A. Siemko, A. Verweij, and B. Vullierme
}

\begin{abstract}
In the framework of the series tests of superconducting magnets for the LHC, a special procedure was developed at CERN to perform conductor limited quenches at temperatures around 4.4 K. All results obtained on pre-series and series main dipoles tested to date will be presented with their analysis. These quenches allow fine diagnostics concerning the electrical integrity of the conductors and of the splices. They also allow the determination for each magnet of the temperature margin at nominal operating conditions of the LHC at superfluid helium. The comparison between the quench current and the critical current directly measured on short samples of superconducting cables used for the winding is discussed.
\end{abstract}

Index Terms-Conductor limited quenches, critical current, large hadron collider, quench current, superconducting magnets.

\section{INTRODUCTION}

$\mathbf{T}$ HE Large Hadron Collider (LHC) currently under construction at CERN required extensive research and development of various technologies, especially those related to superconducting magnets. To maintain protons in orbit at the unprecedented nominal energy level of $7 \mathrm{TeV}$ within the constraint of the $27 \mathrm{~km}$ circumference LEP tunnel, the 1232 LHC main dipoles must provide a magnetic field of $8.33 \mathrm{~T}$ on a length equal to $14.3 \mathrm{~m}$. This is achieved by using NbTi superconducting coils cooled by superfluid Helium at $1.9 \mathrm{~K}$ and a proper mechanical design to sustain the resulting Lorentz force [1].

Like most large superconducting magnets, LHC main dipoles and quadrupoles exhibit training quenches at $1.9 \mathrm{~K}$, i.e. a progressive increase of the current level reached after repeated quenching. These quenches originate from conductor motion [2] and the experience gained at CERN with extensive studies on 1-meter long and full-scale dipole prototypes has demonstrated that when the magnet powering is limited to current values around the $4.4 \mathrm{~K}$ short sample limit of the conductors, the mechanical activity of the winding is much reduced. To further stabilize the coil of LHC main dipoles, their training was accomplished at $1.9 \mathrm{~K}$ before the conductor limited quenches around $4.4 \mathrm{~K}$. The results are analyzed by comparing the quench values with the critical current measured on short samples of the superconducting cable of the winding. This investigation has been systematically carried out at the beginning of the production of LHC pre-series dipoles to validate the integrity and in-situ performance of the conductors and also to estimate the temperature margin distribution among

Manuscript received October 5, 2004

The authors are with the European Laboratory for Nuclear Research, CERN, CH-1211 Geneva 23, Switzerland (e-mail: Pierre.Pugnat@cern.ch).

Digital Object Identifier 10.1109/TASC.2005.849168 the main dipoles at nominal operating conditions of the LHC. At present, quenches around $4.4 \mathrm{~K}$ are mainly performed on magnets showing insufficient performance at $1.9 \mathrm{~K}$. Combined with a precise localization of the beginning of the transition using quench antennas [3], these quenches allow to detect and to identify possible degradation of the conductor performance or faulty electrical connections between the superconducting cables of the inner and the outer layers [4]. Precise diagnostics of the quench origin of the main superconducting magnets is an important step in the quality control. Any drift or critical fault in the manufacturing process must be detected and precisely identified to allow the manufacturers to take appropriate corrective actions.

In the first part of this article, the experimental procedure for the quenches performed around $4.4 \mathrm{~K}$ will be described including the magnet current pre-cycle performed at $1.9 \mathrm{~K}$ to stabilize the coil, the cryogenic process to change the magnet temperature from $1.9 \mathrm{~K}$ to $4.4 \mathrm{~K}$ and the various type of powering cycles performed at $4.4 \mathrm{~K}$ up to the quench. In a second part, all results obtained and analyzed to date will be presented together with illustrative examples concerning LHC superconducting dipoles with an anomalous behavior.

\section{EXPERIMENTAL}

\section{A. Mechanical Stabilization of the Coil at $1.9 \mathrm{~K}$}

The conductor limited quenches are performed at temperatures around $4.4 \mathrm{~K}$, after the successful training of the magnet at $1.9 \mathrm{~K}$ up to the ultimate field of the LHC equal to $9 \mathrm{~T}$. This step is particularly important to improve the mechanical stability of the winding and allows, typically, a reproducibility of the quench current better than $0.1 \%$ whereas the uncertainty in the current measurements is typically in the $10 \mathrm{ppm}$ range.

\section{B. Cryogenic Processes and Temperature Measurements}

A short description of the CERN Superconducting Magnet Test Plant (SMTP) is given in [2]. For this study, cryo-dipoles were connected to Cryogenic Feeder Units (CFU) which are thermally insulated vessels containing the helium pipe work and helium-cooled high-current circuits [5]. On test benches, the cooling of the magnet is performed first by using helium gas, from room temperature to about $80 \mathrm{~K}$, and then with liquid helium (LHe). At $4.4 \mathrm{~K}$ the magnet is filled with about 300 liters of LHe and is cooled down to $1.9 \mathrm{~K}$ by pumping on the bath in the gas-liquid heat exchanger tube, which extracts the heat from the cold mass all along its length. The magnet warm-up from $1.9 \mathrm{~K}$ to $4.4 \mathrm{~K}$ used to be obtained by stopping the pumping of the heat exchanger tube. However, this slow warm-up process 


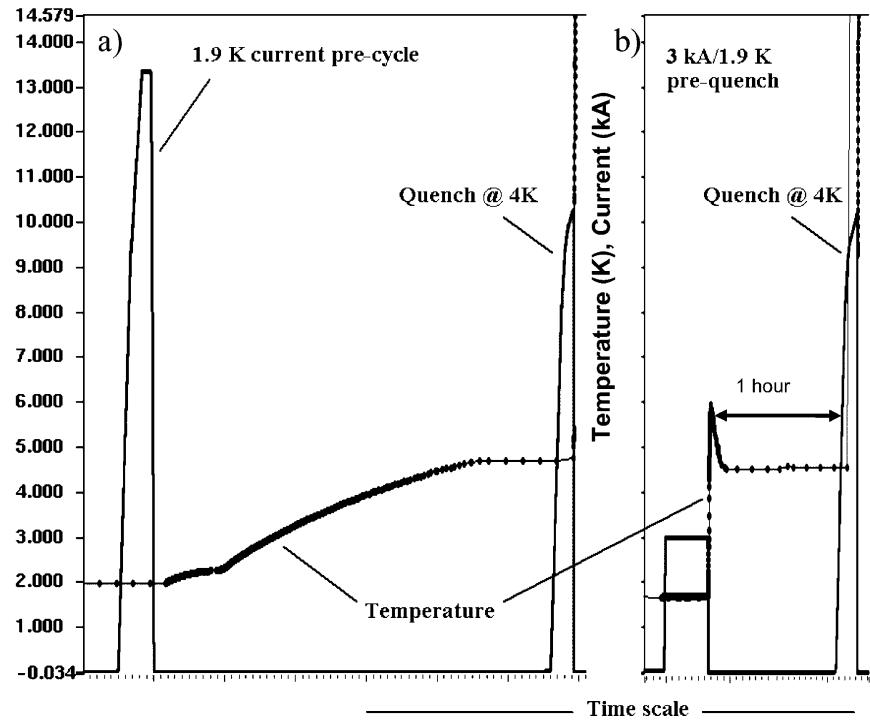

Fig. 1. Procedures for the magnet temperature change from $1.9 \mathrm{~K}$ to $4.4 \mathrm{~K}$. In a) the magnet warm-up is naturally driven by the heat in-leak, in b) a $3 \mathrm{kA}$ provoked quench produces an accelerated warm-up.

mainly driven by the cryo-magnet heat in-leak took about 3 hours (Fig. 1). To reduce the time to less than one hour in the framework of series tests, a different procedure was developed. A quench at $3 \mathrm{kA}$ was provoked with quench heaters to produce the global heating that is just sufficient to increase the magnet temperature up to $4.4 \mathrm{~K}$ (Fig. 1).

The study of conductor limited quenches of a superconducting magnet requires an accurate determination of the temperature of the coil. For the data presented in this study, three inputs were used, two coming from Cernox temperature sensors and one from the helium gas pressure measured above the bath. One of the temperature sensors was located at the middle of the cold mass and the other one at the outlet of the magnet LHe supply. Both were calibrated to guarantee a measurement uncertainty within $\pm 10 \mathrm{mK}$ [1]. A detailed study has shown a systematic difference of about $23 \pm 5 \mathrm{mK}$ between the temperature determined from the pressure measurement and the one given by the thermometers. This corresponds, taking into account the accuracy of the sensors, to the hydrostatic pressure of the LHe column inside the CFU. Conductor limited quenches were performed in stable pressure and temperature conditions within an overall uncertainty of $\pm 15 \mathrm{mK}$.

\section{Magnet Powering Cycles}

In this article, Conductor Limited Quenches (CLQ) of LHC main dipoles performed around $4.4 \mathrm{~K}$ refer to Short-Sample Limit Quench (SSLQ), Direct Ramp to Quench (DRQ) and V-Ramp to Quench (VRQ). SSLQ have been performed at a magnet current ramp-rate $d I / d t=2 \mathrm{~A} / \mathrm{s}$. DRQ have been carried out with current ramp-rate up to $80 \mathrm{~A} / \mathrm{s}$. To interpret the ramp-rate effects on the quench current, special VRQ current cycles were developed. The magnet is powered at fixed $d I / d t$ up to the DRQ current value minus 100 A for a plateau of 15 minutes. The current is then ramped down to $50 \mathrm{~A}$ before being ramped up to the quench at the same absolute value of $d I / d t$ (V-shape).

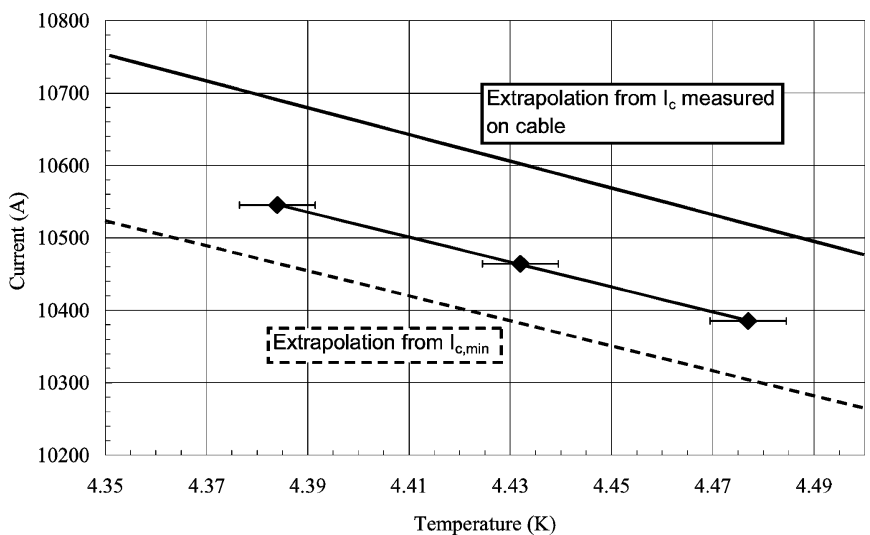

Fig. 2. Results of conductor limited quenches performed on the dipole 1002. Two quenches were performed at $4.385 \mathrm{~K}$ with a current difference of $1 \mathrm{~A}$.

\section{RESULTS AND ANALYSIS}

\section{A. SSLQ and Comparison With $I_{c}$}

Fig. 2 gives an example of the quench currents obtained in a pre-series dipole as a function of the measured temperature. The upper line represents the extrapolated critical current $I_{c}$ measured for each dipole on a short sample of the cable used for the winding [6] with a typical uncertainty of $\pm 2 \%$. The lower line corresponds to the extrapolated minimum specification of the inner cable critical current, $I_{c, \min }$, equal to $14140 \mathrm{~A}$ at 4.222 $\mathrm{K}$ in 7T field [6]. Conductor limited quenches generally occur (i.e. in absence of any defect) in the first turn of the inner layer being subject to the peak field of the coil, $3 \%$ higher than the central field [1].

The comparison of the quench current $I_{q}$ with $I_{c}$ and $I_{c, \min }$ must be performed at the same magnetic field and temperature. For this purpose, the following formula was used:

$I_{c}\left[T_{q}, B_{q}\right]=\left(I_{c}[4.22 \mathrm{~K}, 7 \mathrm{~T}]-s \cdot\left(B_{q}-7\right)\right) \cdot \frac{T_{c}\left(B_{q}\right)-T_{q}}{T_{c}\left(B_{q}\right)-4.22}$

where $T_{q}$ is the temperature of the quench, $B_{q}$ the magnetic field given by the transfer function measured on each magnet and corrected by the peak field coefficient. The slope $s$ of the $I_{c}[4.22 \mathrm{~K}, B]$ variation was deduced from two measurements of $I_{c}$ at 6 and $7 \mathrm{~T}$ and $T_{c}(B)$ expresses the field dependence of the critical temperature (inverse Lubell formula):

$$
T_{c}(B)=T_{c 0} \cdot\left(1-\frac{B}{B_{c 20}}\right)^{\frac{1}{1.7}}
$$

with for $\mathrm{NbTi}, T_{c 0}=9.2 \mathrm{~K}$ and $B_{c 20}=14.5 \mathrm{~T}$.

The relative difference measured between quench current and critical current are given in Fig. 3 for the first 39 LHC dipoles cold-tested and delivered by the three manufacturers. Results are summarized in Table I and the following comments can be made before discussion:

i) A trend to have a systematic and significant larger values for $I_{c}$ with respect to $I_{q}$ is observed.

ii) The results obtained can be interpreted as being manufacturer dependant.

Dipoles coming from manufacturer 02 will not be considered in the following discussion because of the poor statistics. More 

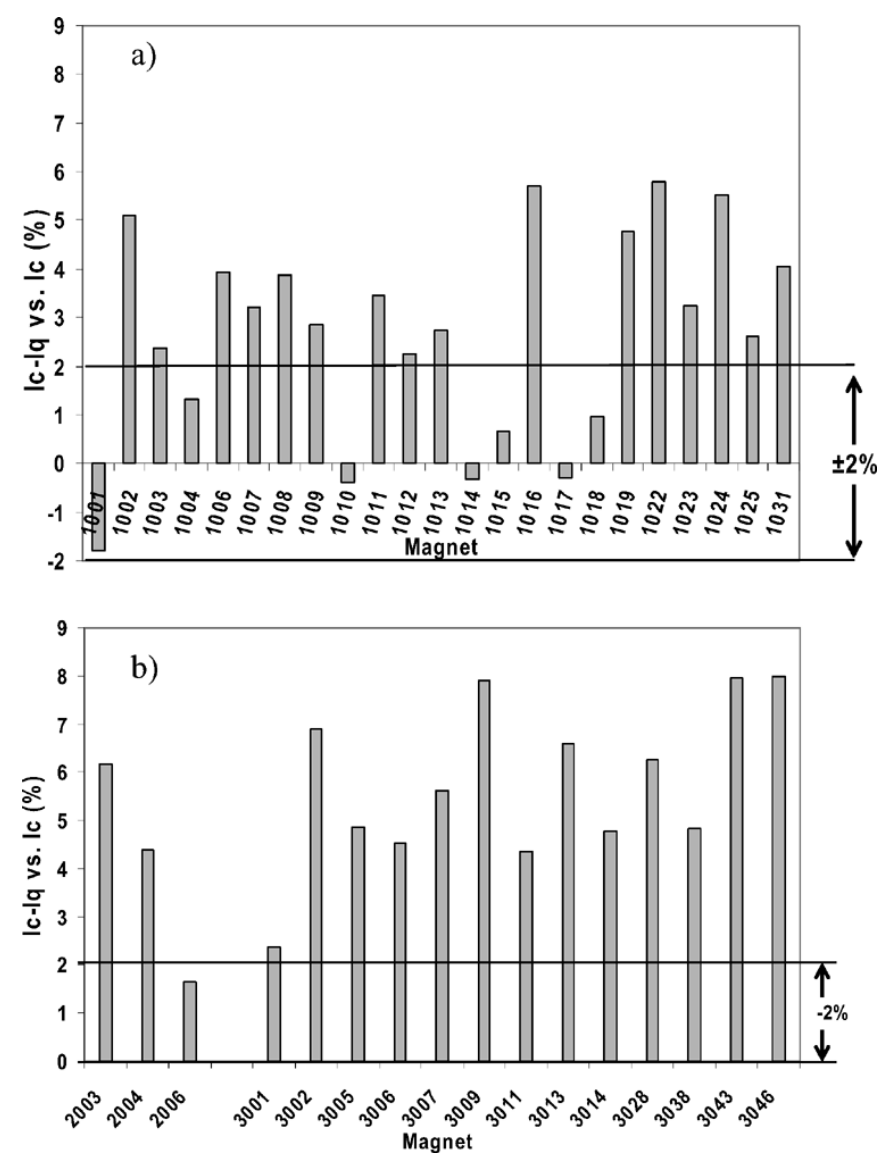

Fig. 3. Relative difference between $I_{c}$ and $I_{q}$ for superconducting dipoles produced by the manufacturer 01 in a) and by manufacturers 02 and 03 in b). Each bar was obtained from an average of 2, 3 or 4 measured $I_{q}$-values.

TABLE I

RESUlTS FOR THE THREE COLD MASS PRODUCERS

\begin{tabular}{clccc}
\hline \hline & Producer & 01 & 02 & 03 \\
\hline & Average & 2.68 & $(4.07)$ & 5.77 \\
& Stand.Dev. of Av. & 0.44 & $(2.62)$ & 0.47 \\
& Stand.Dev. & 2.13 & $(2.28)$ & 1.69 \\
& Min & -1.79 & 1.65 & 2.38 \\
& Max & 5.79 & 6.17 & 7.99 \\
\hline \multirow{0}{*}{} & Average & 1.53 & $(1.54)$ & 1.52 \\
& Stand.Dev. & 0.04 & $(0.01)$ & 0.06 \\
& Min & 1.46 & 1.53 & 1.38 \\
& Max & 1.62 & 1.55 & 1.59 \\
\hline \hline
\end{tabular}

Data between parentheses have poor statistics.

results for this manufacturer showing an unusual quench behavior will be discussed in the paragraph $\mathrm{C}$ below.

To interpret point i), the difference in experimental conditions must be considered. Short sample $I_{c}$ is defined as the current for which the resistivity of the superconductor exceeds $10^{-14} \Omega \mathrm{m}$, while the magnet $I_{q}$ is a thermal runaway current, defined at the quench detector threshold equal to $200 \mathrm{mV}$. For short-sample limit quenches around $4.4 \mathrm{~K}$, the propagation velocity measured in peak field is typically around $60-70 \mathrm{~m} / \mathrm{s}$ and the threshold is in general reached in no more than $5 \mathrm{~ms}$ after the first signature of the transition detected with quench antennas. With a current ramp-rate of $2 \mathrm{~A} / \mathrm{s}$, this brings a negligible current correction. For short-sample $I_{c}$ measurements, a larger ramp-rate of

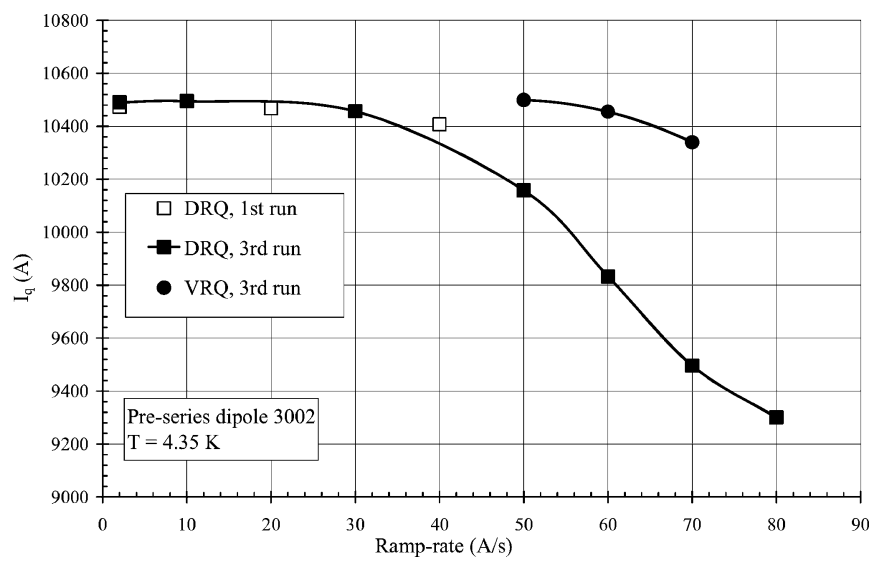

Fig. 4. Quench current as a function of the ramp-rate $d I / d t$.

$150 \mathrm{~A} / \mathrm{s}$ is applied but no effect of the eddy currents induced by the self-field was detected on the measurement results. Differences in cryogenic conditions must also be mentioned between $I_{c}$ and $I_{q}$ measurements, as well as, the difference in the magnetic field distribution. Inside the dipole, the cables are subjected to a field gradient of $1.13 \mathrm{~T} / \mathrm{cm}$, whereas for $I_{c}$ measurements, the nonuniformity only comes from the self-field distribution. All the above mentioned differences in experimental conditions could explain the systematic difference between $I_{c}$ and $I_{q}$. A different explanation must be sought to interpret point ii) i.e. the significant difference between the averaged values (Table I) related to superconducting dipoles assembled by companies 01 and 03. This result supports an interpretation that considers a degradation of the in-situ performance of the superconducting cables with respect to the $I_{c}$ measurements obtained before the winding of coils. This degradation is found to depend on the cold mass manufacturer. Further investigations could be launched to identify the origin and relevant aspects of the manufacturing process, which are most likely to influence the conductor's performance. Differences among manufacturers in the control of the parameters of the curing process of coil poles could constitute a part of the explanation.

\section{B. Special Investigations With DRQ and VRQ at $4.4 \mathrm{~K}$}

DRQ and VRQ allow further fine investigation of specific problems inside magnets such as, for example, the distinction between abnormal heating and a nonuniform splice resistance distribution. To interpret results and to determine the amplitude of nonuniform current distribution with long time constants in some reference cases, this experimental investigation has been associated to local field measurements using Hall plates [7], [8]. Effects of Boundary Induced Coupling Currents (BICC) were extensively studied at CERN [9] and offer an explanation for the difference between DRQ and VRQ results. Fig. 4 shows a typical example of the effect of the powering history on the quench currents. VRQ gives a larger $I_{q}$ mainly because the V-precycle reduced the BICC whereas the heating due to uniform current distribution is nearly the same in both cases [7]-[9]. One of the main parameters needed to interpret these results is the cross-contact resistance $R_{c}$ between strands of the Rutherford cable. Field advance measurements performed with 
coils and Hall plates as a function of $d I / d t$ give, for dipoles 3001 and 3002, an average $R_{c}$ around $100 \mu \Omega$.

\section{Special Cases}

Several magnets delivered by company 02 showed abnormal quench results at $4.4 \mathrm{~K}$. One of them was obtained from VRQ whereas all other from SSLQ. All these quenches were initiated at approximately the same location and voltage taps signals displayed unusual shapes at the beginning of the resistive transition. The position of the quenches was approximately at $400-500 \mathrm{~mm}$ from the connection-side end where the field is reduced by about $9 \%$ due to the use of nested laminations. These results suggest a damaged conductor or a faulty splice connecting the inner and the outer layer cables. Because these faults have practically no influence on the magnet operational quench performance, these superconducting dipoles were accepted by CERN and will be used at special locations in the LHC tunnel where the beam loss is smaller. On the other hand, investigation carried out in industry during the coil assembly process allowed to identify a problem with the tooling used to form the splices and to define corrective actions.

\section{Algorithm For the Temperature Margin Estimate}

The temperature margin $\Delta T_{S}$ of a superconducting magnet is in general defined at nominal current $\left(I_{n}\right)$ as the difference between the current sharing temperature of the conductor in the design peak field $\left(B_{p}\right)$ and the bath temperature $\left(T_{b}\right)$. It can be expressed as:

$$
\Delta T_{s}\left[T_{b}, B_{p}\right]=\left(T_{c}\left(B_{p}\right)-T_{b}\right) \cdot\left(1-\frac{I_{n}}{I_{c}\left[T_{b}, B_{p}\right]}\right)
$$

where $T_{c}(B)$ is given by (2), $I_{n}=11850 \mathrm{~A}, T_{b}=1.9 \mathrm{~K}$ and $B_{p}=8.58 \mathrm{~T}$. To estimate $\Delta T_{S}$ from conductor limited quenches performed at $4.4 \mathrm{~K}$, several hypothesis have to be made. Firstly, $I_{q}\left[T_{q}, B_{q}\right]$ is considered as the relevant estimator of the in-situ $I_{c}\left[T_{q}, B_{q}\right]$. Then by applying (1) to the result of conductor limited quenches, $I_{c}[4.22 \mathrm{~K}, 7 \mathrm{~T}]$ can be deduced. Secondly, as for each cable producer the ratio $I_{c}[1.9 \mathrm{~K}, 10 \mathrm{~T}] / I_{c}[4.2 \mathrm{~K}, 7 \mathrm{~T}]$ was measured and found to be stable within $0.1 \%$, the in-situ value for $I_{c}[1.9 \mathrm{~K}, 10 \mathrm{~T}]$ can be calculated. Thirdly, $I_{c}[1.9 \mathrm{~K}, 8.33 \mathrm{~T}]$ can be estimated, from the measurements of $\mathrm{d} I_{c} / d B$ performed at $1.9 \mathrm{~K}$ on cables and found to be stable for each producer.

The temperature margins of dipoles produced by each company were deduced from conductor limited quenches using (3).
The average and min-max values are reported in Table I. All but one $\Delta T_{S}$ values are larger than the $1.4 \mathrm{~K}$ minimum design parameter, estimated for the inner cable to balance all terms contributing to the heat generation at nominal conditions of the LHC, including beam losses [1]. The temperature margin gives, in addition to training quenches, complementary information for the performance evaluation of each dipole and can be used to sort and position the magnets in the LHC to optimize their performance.

\section{CONCLUSION}

Conductor limited quenches at $4.4 \mathrm{~K}$ allow a fine in-situ quality control of the conductor and connection integrity inside LHC superconducting main dipoles. They have revealed abnormal behavior of magnets of one manufacturer, which were traced back to the tooling used to form the splices. In addition, conductor limited quenches at $4.4 \mathrm{~K}$ allow to estimate the temperature margin of the LHC dipoles in working conditions, taking into account possible conductor degradations occurring during the cold mass manufacturing process.

\section{ACKNOWLEDGMENT}

The authors thank the operation and the support teams involved in the tests of LHC superconducting magnets at CERN.

\section{REFERENCES}

[1] The LHC Study Group, "The Large Hadron Collider Design Report,", CERN-2004-003, vol. I, Jun. 4, 2004.

[2] A. Siemko et al., "Quench performance and field quality of the LHC pre-series superconducting dipoles," IEEE Trans. Appl. Supercond., vol. 14, no. 2, pp. 165-168, 2004.

[3] D. Leroy and J. Krzywinski et al., "Quench observation in LHC superconducting one meter long dipole models by field perturbation measurements," IEEE Trans. Appl. Supercond., vol. 3, pp. 781-784, 1993.

[4] P. Pugnat, "Spot-Check of Splice Resistance,", unpublished, CERN/EDMS 369868.

[5] V. Benda et al., "Experience with a pre-series superfluid helium test bench for LHC magnets," in Proc. ICEC18, Mumbai, India, 2000.

[6] J. D. Adams et al., "Status of the LHC Superconducting cables mass production," IEEE Trans. Appl. Supercond., vol. 12, no. 1, pp. 1056-1062, 2002.

[7] P. Pugnat, T. Schreiner, and A. Siemko, "Investigation of the periodic magnetic field modulation in LHC superconducting dipoles," IEEE Trans. Appl. Supercond., vol. 12, no. 1, pp. 258-261, 2002.

[8] T. Schreiner, "Current Distribution Inside Rutherford-Type Superconducting Cables and Impact on Performance of LHC Dipoles," Ph.D. thesis, Vienna Univ. Technol., 2002.

[9] A. Verweij, "Electrodynamics of Superconducting Cables in Accelerator Magnets," Ph.D. thesis, Univ. Twente, The Netherlands, 1995. 